\title{
Um Estudo de Predição de Volumes de Clones de Eucalipto Utilizando Redes Neurais Artificiais
}

\author{
Welington G. Rodrigues* Fabrizzio A. A. M. N. Soares* \\ Christian D. Cabacinha ** Rogerio Salvini* Gabriel Vieira * \\ Cristhiane Gonçalves* Thyago P. Carvalho* \\ Deborah S. A. Fernandes Carvalho*
* Instituto de Informática e Instituto de Física, Universidade Federal de Goiás - Goiânia, GO, Brazil
** Instituto de Ciências Agrárias, Universidade Federal de Minas
Gerais - Montes Claros, MG, Brazil

\begin{abstract}
Forest inventory is an important element for the effective management of forest resources. Through it is possible, for example, to quantify trees, identify species of a settlement and obtain the total volume to be explored. Volume is one of the most important elements for the exploration of a given area. Therefore, there is the challenge of methods that can precisely calculate the volume of trees without raising costs, including the use of artificial neural networks. This paper aims to present an approach with artificial neural networks for diameter prediction and volume calculation of eucalyptus clones. Models were proposed with and without total tree height, which is costly to obtain in the field. The results achieved were quite promising in relation to traditional methods, besides minimizing the parameters used for the estimation of volumes, thus presenting a path for forest inventory automation.

Resumo: O inventário florestal é um elemento de grande importância para o gerenciamento efetivo dos recursos florestais. Através dele é possível, por exemplo, quantificar árvores, identificar as espécies de um povoamento e obter o volume total a ser explorado. $\mathrm{O}$ volume constitui um dos elementos mais importantes para a exploração de uma determinada área. Portanto, existe o desafio de métodos que possam calcular precisamente o volume das árvores sem elevar os custos, dentre eles, o uso de redes neurais artificiais. Este trabalho tem por objetivo apresentar uma abordagem com redes neurais artificiais para a predição de diâmetros e cálculo do volume de clones de eucalipto. Foram propostos modelos com e sem a altura total da árvore, medida essa onerosa de se obter em campo. Os resultados alcançados mostraram-se bastante promissores em relação aos métodos tradicionais, além de minimizar os parâmetros utilizados para a estimativa dos volumes apresentando, deste modo, um caminho para automação do inventário florestal.
\end{abstract}

Keywords: Multilayer Perceptron; Neural Networks; Forest inventory; Estimating diameter. Palavras-chaves: Multilayer Perceptron; Redes Neurais; Inventário Florestal; Predição de Diâmetros.

\section{INTRODUÇÃO}

O setor florestal brasileiro é mundialmente reconhecido pela sua produtividade, o tempo entre o plantio e a colheita representa a menor rotatividade conhecida. A área total de árvores plantadas no Brasil totalizou 7,84 milhões de hectares em 2016, resultando em um crescimento de 0,5\% em relação a 2015, devido exclusivamente ao aumento das áreas com eucalipto. Dados mostram que os plantios de eucalipto ocupam 5,7 milhões de hectares da área de árvores plantadas do país, além de ser responsável por $91 \%$ de toda a madeira produzida para fins industriais e $6,2 \%$ do PIB Industrial brasileiro (IBÁ, 2017). Esse crescimento deve-se principalmente ao investimento de indústrias que utilizam cada vez mais o eucalipto como matéria-prima para sua produção. Os plantios de eucalipto ocupam 5,7 milhões de hectares da área de árvores plantadas do país, já os plantios da espécie pinus ocupam 1,6 milhão de hectares. Em 2016, o país esteve na liderança global de produtividade florestal, com uma média de $35,7 \mathrm{~m} /$ ha ao ano para os plantios de eucalipto e $30,5 \mathrm{~m} /$ ha ao ano para os plantios de pinus (IBÁ, 2017).

Dentre as métricas utilizadas no inventário florestal, o cálculo do volume de madeira plantado é uma informação essencial no direcionamento racional e sustentável dos recursos disponíveis nas florestas. Portanto, é necessário quantificá-lo de maneira precisa. Para isto, são necessárias diversas medições em amostras representativas das florestas para se conhecer dinâmica dos povoamentos (Soares et al., 2011a). Além das equações volumétricas para cálculo de volume (SCHUMACHER, 1933), o método de cubagem rigorosa permite a mensuração de maneira mais precisa. 
No entanto, uma desvantagem consiste na necessidade de abate de árvores selecionadas. Além de ser um processo lento e implica a comercialização imediata para evitar perder o produto (Cabacinha, 2003). Os algoritmos e métodos estatísticos buscam estabelecer as relações não lineares para calcular o volume em função do diâmetro e altura, a fim de garantir maior eficiência na modelagem.

Neste trabalho são apresentados três diferentes modelos de redes neurais com diferentes parâmetros de entrada para o cálculo de volume de clones de eucalipto. A rede MLP1 é um modelo recursivo que utiliza como entrada os diâmetros $d_{0.3}, d_{0.7}$ e $d_{1,3 m}$ e estima os diametros seguintes de $1 \mathrm{em} 1 \mathrm{~m}$ até a altura total da árvore, posteriormente o volume é calculado através do método de Smalian. A MLP2 consiste em uma rede neural que utiliza como entrada os mesmos diâmetros da MLP1, porém sem a necessidade do conhecimento prévio da altura da árvore. Por fim, a MLP3 utiliza o DAP e a altura total $(H t)$, uma adaptação do modelo de Schumacher e Hall (log), porém sem a necessidade dos parâmetros $\beta^{\prime} s$ e o $\varepsilon$.

O trabalho está organizado da seguinte maneira: A seção II descreve uma abordagem sobre o uso de redes neurais no âmbito florestal, existentes na literatura. A seção III apresenta sobre os métodos de predição e as arquiteturas de Multilayer Perceptron utilizadas neste trabalho. Os materiais e métodos utilizados são descritos na seção IV. A seção $\mathrm{V}$ discorre sobre os resultados alcançados pelos modelos e os compara com os métodos tradicionais. Por fim, a seção VI apresenta as conclusões geradas pelo trabalho.

\section{REVISÃO DA LITERATURA}

As principais vantagens do uso de redes neurais em diversos problemas consiste na capacidade de aproximar funções. Muitos desses atributos possuem relações não lineares, assim, as redes neurais apresentam ótimas opções para analisar esse problema.

Xiao et al. (1998) utiliza redes neurais de múltiplas camadas para estimar a altura de árvores utilizando dados de alta frequência, obtidos através de instrumentos de interferometria. Leduc et al. (2001) faz uso de redes neurais para estimar diâmetros de árvores da espécie Pinus. Diamantopoulou (2005) apresenta uma arquitetura de rede neural para estimar diâmetros e calcular o volume de árvores do gênero Pinus. Os resultados obtidos neste trabalho apresentam a rede neural como uma excelente alternativa aos métodos de regressão. Huang et al. (2009) utiliza uma rede neural de para estimar a frequência dos diâmetros de árvores do gênero Pinus. Marques da Silva et al. (2009) realiza ajuste do modelo de Schumacher e Hall para estimar volume de eucaliptos através de redes neurais.

Niska et al. (2010) utilizou redes neurais para predição de espécies e cálculo de volumes de árvores de três diferentes tipos através varreduras de laser e imagens aéreas. Özçelik et al. (2010) redes neurais foram utilizadas para cálculo de volume de fustes de árvores de quatro diferentes espécies. Diamantopoulou (2010) realiza o tratamento de lacunas de diâmetros com a árvore em pé. Dados faltantes são essenciais para uma boa precisão no inventário florestal.
Guo et al. (2011) mostrou que redes neurais são eficientes para resolver problemas complexos não lineares como a distribuição de diâmetros.

Soares et al. (2011b) aborda a predição recursiva para estimar diâmetros de clones do eucalipto com e sem o conhecimento prévio da altura da árvore. Binoti et al. (2014) estimam volumes de árvores de eucalipto utilizando o diâmetro à altura do peito $(D A P)$, a altura e os diâmetros nas posições de 0,$0 ; 0,5 ; 1,0 ; 1,5 ; 2,0 ;$ e $4,0 m$ do solo e os volumes obtidos até 2 e $4 m$. Técnicas de redes neurais e lógica fuzzy são utilizadas em Vieira et al. (2018) para estimar o crescimento de florestas de eucalipto, predizendo diâmetro e altura de cada árvore através do $D A P$.

\section{PREDIÇÃO E MULTILAYER PERCEPTRON}

\subsection{Predição}

Em problemas de predição, um modelo é construído dada sua relação entre as entradas e as saídas. Este modelo então é capaz de predizer valores futuros dado os valores anteriores. A predição direta e recursiva são métodos bastante utilizados para prever séries temporais (Ji et al., 2005).

A abordagem de predição direta constrói $M+1$, diferentes modelos para prever valores de uma série,

$$
\hat{d}_{i+m}=f_{m}\left(d_{i-1}, d_{i-2}, \ldots, d_{i-n}\right),
$$

onde $m=0,1, \ldots M$, com $M$ definido como o tamanho máximo do horizonte de predição. A variável $d_{i}$ representa os diâmetros na altura $i$, que formam o regressor, onde $n$ é o tamanho do regressor.

A predição recursiva constrói o modelo realizando inicialmente um passo a frente.

$$
\hat{d}_{i}=f\left(d_{i-1}, d_{i-2}, \ldots, d_{i-n+1}\right),
$$

Diferente da predição direta, próximo valor a ser predito, tem como entrada o resultado dela própria no passo anterior, como pode ser observado na Equação (3).

$$
\hat{d}_{i+1}=f\left(\hat{d}_{i}, d_{i-1}, d_{i-2}, \ldots, d_{i-n}\right)
$$

Este trabalho faz uso de ambos métodos para a construção dos modelos. A predição direta é utilizado para os modelos de rede neural com entradas de $d_{0.3}, d_{0,7}, d_{1.3}$ sem o conhecimento da altura total e para o modelo que possui como variáveis de entrada o $(D A P)$ e a altura total $(H t)$. Já a predição recursiva é utilizada no modelo que prever os próximos diâmetros dado os diâmetros iniciais de $d_{0.3}$, $d_{0,7}$, e $d_{1.3 m}$ com passos de 1 em $1 \mathrm{~m}$ até que se atinja a altura total da árvore.

\subsection{Multilayer Perceptron - MLP}

Um perceptron multicamadas é uma função matemática que mapeia um conjunto de valores de entrada para valores de saída. A função é formada pela composição de muitas funções mais simples (Bengio et al., 2015). 
A Tabela (1) apresenta a estrutura das redes MLPs, as funções de ativação, variáveis de entrada e valores de saída dos modelos utilizadas neste trabalho.

Tabela 1. Variáveis de entrada e saída dos modelos de Redes Neurais.

\begin{tabular}{cccc}
\hline Modelo & Função de & Variáveis de & Variáveis de \\
\hline & Ativação & Entrada & Saída \\
\hline MLP1 & Tanh & $d_{0.3}, d_{0.7}$ e $d_{1.3}$ & $\hat{d}_{n}$ \\
MLP2 & Tanh & $d_{0.3}, d_{0.7}$ e $d_{1.3}$ & $v$ \\
MLP3 & Tanh & $D A P$ e $H t$ & $v$ \\
\hline
\end{tabular}

Diferentes funções de ativação podem ser utilizadas no treinamento das redes MLPs. Devido à capacidade de trabalhar problemas não lineares, neste trabalho foi utilizada a função de tangente hiperbólica conforme a Equação (4).

$$
\varphi=\tanh (a)=\frac{2}{(1+\exp (-2 a))}-1
$$

Existem diversos algoritmos de treinamento eficiente de redes neurais. Muitos trabalhos empregam o método de gradiente descendente estocástico $(S G D s)$ para resolver questões de aprendizado de máquina. Uma das características que o tornam bastante difundido, consiste na fácil implementação e o bom desempenho quando existem muitos dados para o treinamento.

Os métodos Quasi-Newton apresentam-se como uma solução de acelerar a técnica de descida do gradiente para a minimização de funções (Shanno, 1970). Nos métodos Quasi-Newton, a matriz Hessiana são é calculada, em vez disso, elas são estimadas por meio de avaliações aproximadas do gradiente.

Os L-BFGS constituem métodos poderosos para resolver problemas onde as matrizes Hessianas não podem ser computadas com um custo razoável ou são muito densas para serem manipuladas facilmente (Nocedal and Wright, 2006).

Neste trabalho, foi utilizada a IDE Spyder 3, Jupyter notebook e Python 3.5 como ambiente de implementação dos modelos de MLP.

\section{MATERIAIS E MÉTODOS}

\subsection{Dados}

O conjunto de dados obtido para a realização deste trabalho foi cedido por (Soares et al., 2011a). Os dados consistem em 615 árvores obtidas de um sítio florestal de clones de eucalipto de propriedade da Empresa Aracruz Celulose S.A., localizada no município de Aracruz na região metropolitana do estado do Espírito Santo. Estas árvores foram amostradas foram cubadas rigorosamente pelo método de Smalian (Cabacinha, 2003). A Tabela (2) apresenta a distribuição estatística do conjunto de dados.

As medições foram realizadas com suta em diâmetros nas alturas 0,$10 ; 0,30 ; 0,50 ; 0,70 ; 0,90,1,10 ; 1,30 ; 2,00 \mathrm{~m}$ em relação ao solo, no restante do tronco em intervalos de 1 em $1 \mathrm{~m}$ e o diâmetro no topo da árvore é considerado igual a zero $\mathrm{cm}$. O processo de medição pode ser observado na Figura (1).

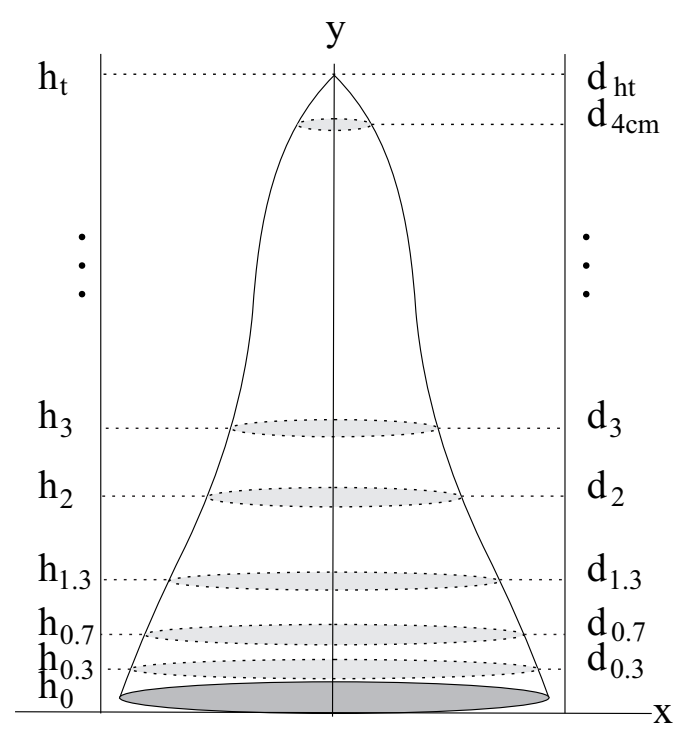

Figura 1. Altura medida e seus diâmetros

Para preparar os dados para as redes perceptron e para que os valores dos diâmetros operem em uma mesma escala, os diâmetros foram normalizados em uma faixa de $[-1,1]$, utilizando a Equação (5)

$$
X^{\prime}=a+\frac{\left(X-X_{\min }\right)(b-a)}{X_{\max }-X_{\min }}
$$

onde $X^{\prime}$ é o valor do diâmetro normalizado, $X$ é o valor do diâmetro atual a ser normalizado, $X_{\max }$ e $X_{\min }$ são os valores do maior e menor diâmetros respectivamente e as variáveis $a$ e $b$ são os valores da faixa de normalização.

\subsection{Cálculo do erro dos diâmetros estimados}

A arquitetura de MLP1 utiliza o método de predição recursiva para estimar os próximos diâmetros dados os diâmetros iniciais até que a altura da árvore seja alcançada. Para verificar a precisão dos diâmetros preditos pela MLP1, foram utilizadas as métricas Root Mean Square Error $\left(\mathrm{RMSE}_{\%}\right)$ e a Correlação Linear $(R)$ conforme as equações 6 e 7 respectivamente (Soares et al., 2011a).

$$
\begin{gathered}
R M S E \%=\sqrt{\frac{1}{N} \sum\left(y_{i}^{a}-y_{i}^{e}\right)^{2}}\left(\frac{100}{\bar{y}_{i}^{a}}\right) \\
R=\frac{\sum_{i=1}^{n}\left(y_{i}^{a}-\bar{y}^{a}\right)\left(y_{i}^{e}-\bar{y}^{e}\right)}{\sqrt{\sum_{i=1}^{n}\left(y_{i}^{a}-\bar{y}^{a}\right)^{2}} \sqrt{\sum_{i=1}^{n}\left(y_{i}^{e}-\bar{y}^{e}\right)^{2}}}
\end{gathered}
$$

onde $y^{a}$ representa o vetor dos diâmetros reais, $y^{e}$ o vetor dos diâmetros estimados e $\bar{y}^{e}$ e $\bar{y}^{e}$ são os valores médios dos vetores estimados dos diâmetros, respectivamente, e $n$ é o número do diâmetro atual.

\subsection{Cálculo do erro dos volumes estimados}

Com o objetivo de analisar a precisão dos volumes calculados pelos modelos, foram utilizados as métricas de Desvio Médio (MAE\% ) Equação (8), Bias (Bias\% ) Equação (9), $R M S E \%$ Equação (6) e a Correlação Linear $(R)$ Equação (7). 
Tabela 2. Estatísticas do conjunto de dados.

\begin{tabular}{|c|c|c|c|c|c|c|c|c|c|c|c|c|c|c|c|}
\hline \multirow[t]{2}{*}{ Classes } & \multicolumn{2}{|c|}{ Limites } & \multirow[t]{2}{*}{$n$. } & \multicolumn{2}{|c|}{$\operatorname{Autocorr}\left(d_{i}\right)$} & \multicolumn{5}{|c|}{$\mathrm{DAP}(\mathrm{cm})$} & \multicolumn{5}{|c|}{ Altura total $(H t) m$} \\
\hline & Menor & Maior & & Min & Máx & Média & Var & $\mathrm{DP}$ & Min & $\operatorname{Max}$ & Média & Var & SP & Min & Máx \\
\hline 1 & 7.15 & 10.15 & 14 & 0.63 & 0.84 & 8.68 & 0.95 & 0.97 & 7.15 & 10.10 & 17.60 & 3.49 & 1.87 & 14.20 & 19.70 \\
\hline 2 & 10.15 & 13.15 & 106 & 0.77 & 0.87 & 12.01 & 0.54 & 0.73 & 10.32 & 13.14 & 22.83 & 2.75 & 1.66 & 18.00 & 26.70 \\
\hline 3 & 13.15 & 16.15 & 302 & 0.78 & 0.89 & 14.62 & 0.68 & 0.82 & 13.15 & 16.14 & 25.74 & 1.57 & 1.25 & 18.40 & 29.40 \\
\hline 4 & 16.15 & 19.15 & 178 & 0.83 & 0.90 & 17.23 & 0.64 & 0.80 & 16.15 & 19.14 & 27.52 & 1.39 & 1.18 & 24.20 & 30.10 \\
\hline 5 & 19.15 & 22.15 & 15 & 0.82 & 0.90 & 20.21 & 1.7 & 1.31 & 19.31 & 24.55 & 29.23 & 1.06 & 1.03 & 27.20 & 30.60 \\
\hline
\end{tabular}

$$
\begin{aligned}
& M A E_{\%}=\frac{1}{n} \sum_{i=1}^{n}\left(\frac{\left|y_{i}^{e}-y_{i}^{a}\right|}{y_{i}^{a}}\right) 100 \\
& \text { Bias } \%=\frac{\sum_{n=1}^{n} y_{i}^{e}-\sum_{n=1}^{n} y_{i}^{a}}{\sum_{n=1}^{n} y_{i}^{a}} 100
\end{aligned}
$$

onde $y^{a}$ e $y^{e}$ representam os vetores dos volumes reais e estimados, respectivamente e $n$ é o número do volume atual.

\subsection{Cálculo do volume das árvores através de equações volumétricas}

O volume real de todas as árvores do sítio e o volume calculado a partir das estimativas realizadas pelos modelos foram obtidas através do método de Smalian conforme as Equações (10) e (11).

$$
\begin{gathered}
v=\sum_{i=1}^{n-1} \frac{g_{i}+g_{i+1}}{2} l_{i}+\frac{g_{n} l_{n}}{3} \\
g_{i}=\frac{\left(d_{i} / 2\right)^{2} \pi}{10.000}
\end{gathered}
$$

onde $v$ é o volume total da árvore, $g_{i}$ é a área basal na $i$-ésima posição, $l_{i}$ é o comprimento da seção na $i$-ésima posição, $g_{n}$ é a área basal do cone da árvore, $l_{n}$ é o comprimento do cone e $d_{i}$ é o diâmetro da $i$-ésima posição.

Os respectivos volumes das árvores reais e os volumes estimados pela MLP foram comparados com o método de Schumacher e Hall (log), um modelo volumétrico bastante difundido no campo florestal que calcula o volume através das medidas $D A P$ e altura total $(H t)$ conforme a Equação (12). Esse modelo é ajustado com os parâmetros obtidos de árvores cubadas rigorosamente (Cabacinha, 2003).

$$
\ln V=\beta_{0}+\beta_{1} \ln D A P+\beta_{2} \ln H_{t}+\varepsilon
$$

onde v é o volume, $D A P$ é o diâmetro na altura do peito, $\beta 0, \beta 1$ e $\beta 2$ são os parâmetros a serem ajustados e $H t$ é a altura total da árvore.

A análise de variância (ANOVA) de 2-critérios foi o método utilizado para verificar as diferenças estatísticas entre os modelos de MLPs e o modelo de Shucmarcher e Hall $(\log )$. Os testes foram realizados considerandos o valor de $p=0,05$.

\section{RESULTADOS E DISCUSSÃO}

A escolha dos parâmetros das MLPs foi realizada através de busca exaustiva em grade GridSearch, onde foram submetidos ao grid: número de camadas ocultas, funções de ativação, ciclos ou épocas e algoritmos de treinamento. Escolhas aleatórias de parâmetros e técnicas de AutoLearn também foram utilizadas até encontrar os hyperparâmetros que obtivessem os melhores resultados (Bergstra and Bengio, 2012). A escolha final dos parâmetros considerou o menor erro quadrático médio (MSE), visto que adição de novas camadas não trouxeram mudanças significativas no valor do mesmo. O processo de treinamento consistiu em separar o conjunto de treino e teste em $70 \%$ e $30 \%$, respectivamente. Diversos testes foram realizados nos modelos de MLPs apresentados na seção anterior. A MLP1 mostrou resultados significativos com 10 neurônios na camada intermediária. De modo semelhante às redes MLP2 e MLP3 apresentaram bons resultados com 30 neurônios na camada intermediária.

\subsection{Predição de Diâmetros}

Conforme mencionado na seção 3, a arquitetura MLP1 realiza a predição dos diâmetros seguintes dados os diâmetros iniciais nas alturas $0.3,0.7$ e $1.3 \mathrm{~m}$ até que a altura da árvore seja alcançada. Posteriormente o volume é calculado através do método de Smalian e comparada com as demais arquiteturas e o modelo de Schumacher e Hall (log). Os resultados podem ser observados de acordo com a Tabela (4).

Como pode ser observado, a classe 1 apresentou o maior valor de erro RMSE, de $23,5 \%$, que por só é um valor bastante alto. A baixa quantidade de dados para o treinamento da MLP para essa classe, representa um dos principais motivos deste valor. No entanto, a maior parte dos valores de $R M S E_{\%}$ ficou distribuído torno de $5 \%$, o que indica que os demais diâmetros foram estimados com maior precisão. As classes 2, 3 e 4 que representam as árvores mais numerosas, possuem os valores mais altos $R M S E_{\%}$ e $R$ muito próximos, variando em torno de $19 \%$ a $22 \%$ e $97 \%$ a $99 \%$ respectivamente. Já os menores valores de $R M S E_{\%}$ dessas classes ficaram em torno de $2 \%$, apresentando uma correlação bastante forte entre os valores reais e os valores estimados.

A comparação entre os diâmetros reais e estimados no melhor e pior caso árvores da classe 3 podem ser observados na Figura (2). A linha contínua representa os diâmetros reais obtidos através do processo de cubagem, já a linha tracejada representa os valores estimados pela MLP.

A Figura 2(b) mostra a maior parte das árvores do conjunto se encontram nos menores valores de RMSE, o que pode ser entendido como a precisão do modelo sendo satisfatória. 
Tabela 3. Comparação entre os volumes reais e os estimados pelas MLPs.

\begin{tabular}{|c|c|c|c|c|c|c|c|c|c|c|c|c|c|c|c|c|}
\hline \multirow[t]{2}{*}{ Classe } & \multicolumn{4}{|l|}{ MLP1 } & \multicolumn{4}{|l|}{ MLP2 } & \multicolumn{4}{|l|}{ MLP3 } & \multicolumn{4}{|c|}{ Shumacher e Hall (log) } \\
\hline & $\mathrm{MAE}_{\%}$ & Bias\% & $\mathrm{R}$ & RMSE\% & $\mathrm{MAE}_{\%}$ & Bias\% & $\mathrm{R}$ & RMSE\% & $\mathrm{MAE}_{\%}$ & Bias\% & $\mathrm{R}$ & RMSE\% & $\mathrm{MAE}_{\%}$ & Bias \% & $\mathrm{R}$ & RMSE \% \\
\hline 1 & 6.03 & 0.60 & 0.95 & 7.65 & 8.57 & 0.16 & 0.92 & 11.29 & 5.08 & 0.05 & 0.97 & 6.29 & 6.16 & 1.15 & 0.97 & 8.29 \\
\hline 2 & 4.83 & 0.51 & 0.97 & 5.44 & 7.02 & 0.15 & 0.88 & 9.25 & 5.07 & 0.36 & 0.95 & 5.29 & 7.76 & 0.57 & 0.96 & 5.53 \\
\hline 3 & 5.30 & 0.24 & 0.98 & 5.67 & 8.96 & 0.90 & 0.93 & 11.01 & 9.56 & 0.96 & 0.93 & 7.95 & 4.48 & 1.01 & 0.93 & 5.65 \\
\hline 4 & 4.88 & 1.69 & 0.99 & 4.16 & 6.83 & 0.13 & 0.77 & 8.08 & 8.03 & 0.29 & 0.87 & 9.51 & 4.25 & 1.29 & 0.91 & 5.22 \\
\hline 5 & 4.80 & 1.03 & 0.98 & 5.36 & 3.30 & 0.08 & 0.96 & 4.14 & 5.82 & 1.12 & 0.88 & 6.87 & 4.62 & 3.37 & 0.94 & 6.15 \\
\hline
\end{tabular}

Tabela 4. Comparação dos erros de predição de diâmetro por classe.

\begin{tabular}{|c|c|c|c|c|c|c|c|}
\hline \multirow{3}{*}{ Classe } & \multirow{3}{*}{ Qtd } & \multicolumn{6}{|c|}{ MLP1 } \\
\hline & & \multicolumn{4}{|c|}{$R M S E_{\%}$} & \multicolumn{2}{|c|}{ Correlação $R$} \\
\hline & & Min & $\operatorname{Max}$ & Média & $\mathrm{DP}$ & Min & Máx \\
\hline 1 & 14 & 3.83 & 23.50 & 10.28 & 6.26 & 93 & 99 \\
\hline 2 & 106 & 2.17 & 19.21 & 7.02 & 4.36 & 98 & 99 \\
\hline 3 & 302 & 1.67 & 22.59 & 7.00 & 3.63 & 97 & 99 \\
\hline 4 & 178 & 1.51 & 17.66 & 6.65 & 3.26 & 97 & 99 \\
\hline 5 & 15 & 2.39 & 12.93 & 5.06 & 3.07 & 99 & 99 \\
\hline
\end{tabular}

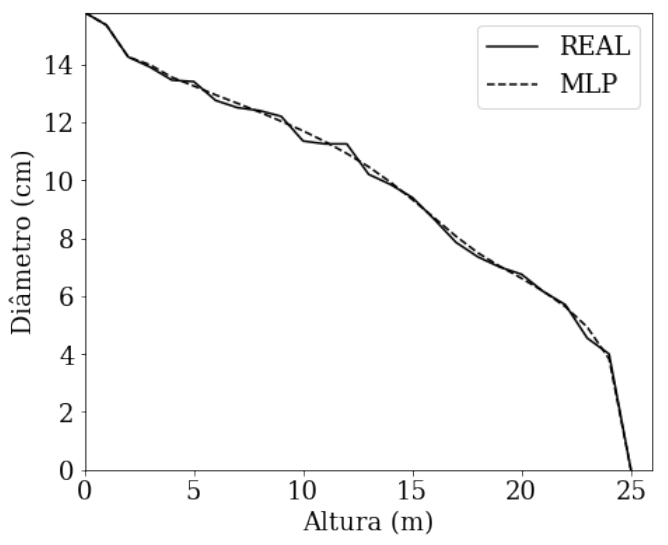

(a) Melhor caso

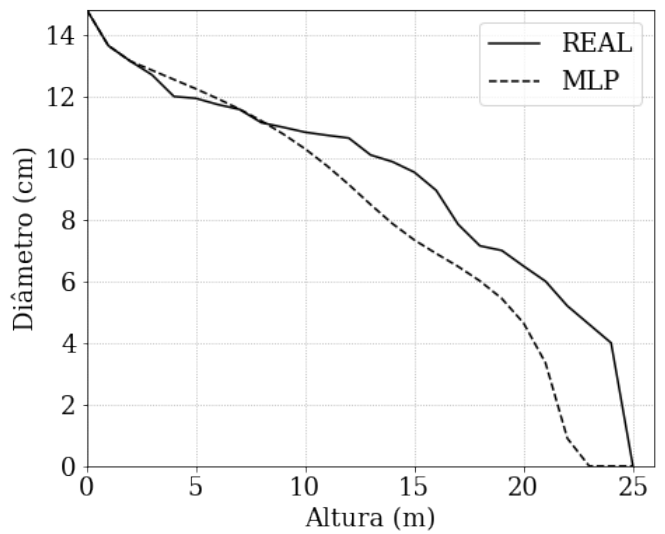

(b) Pior caso

Figura 2. Comparação entre os diâmetros reais (linha contínua) e diâmetros estimados (linha pontilhada) apresentando o melhor e pior caso respectivamente.

5.2 Cálculo dos volumes reais, obtidos pelas MLPs e pelo modelo de Schumacher and Hall (log).

Os valores reais das árvores cubadas foram calculados através o de Smalian, Equação 10. Este mesmo método foi utilizado para calcular o volume dos diâmetros estimados pela MLP1. O método de Schumacher e Hall (log) foi utilizado como medida de comparação, visto que é um modelo bastante empregado no setor florestal. Os parâmetros utilizados para realizar o cálculo de volume foram ajustados pelo trabalho de (Cabacinha, 2003) e aplicados a Equação (12). Os valores correspondentes são: $\beta_{0}=10,79449927$, $\beta_{1}=1,942384069, \beta_{2}=1,226015699$ e $\varepsilon=0$. Com $R^{2}(\%)=99,44$, erro padrão residual $S y x=0,011993 m$, e Syx $\%=5,81$.

As comparações entre os volumes reais calculados pelas MLPs e por Schumacer e Hall (log) podem ser observados na Tabela (5). Os valores apresentados correspondem ao volume total estimado para cada classe.

Tabela 5. Comparação entre os volumes $m^{3}$ reais, calculados pelas MLPs e por Schumacher e Hall $(\log )$.

\begin{tabular}{cccccc}
\hline Classe & Real & MLP1 & MLP2 & MLP3 & $\begin{array}{c}\text { Schumacher } \\
\text { e Hall (log) }\end{array}$ \\
\hline 1 & 0.6560 & 0.6590 & 0.6457 & 0.6563 & 0.6636 \\
2 & 12.7895 & 12.7707 & 12.7692 & 12.7424 & 12.7164 \\
3 & 61.8668 & 61.8655 & 61.8413 & 61.2727 & 61.2559 \\
4 & 53.1030 & 53.1205 & 53.1750 & 53.2596 & 53.7882 \\
5 & 6.4467 & 6.34 & 6.4523 & 6.5191 & 6.6646 \\
\hline Total & 134.862 & 134.7557 & 134.8835 & 134.4501 & 135.0887 \\
\hline
\end{tabular}

Como pode ser observado na Tabela (5), os valores reais e os valores estimados pela MLP são bastante próximos. Embora o método de Schumacher e Hall (log) não apresente diferenças significativas, os valores estimados pelas arquiteturas das MLPs são mais próximas dos valores reais. Esses valores mostram a eficiência das MLPs na estimação de volumes.

Analisando a Tabela (5), percebe-se que a diferença percentual entre os volumes totais reais e os calculados pela MLP1 com predição recursiva, está em torno de 0,07\%, a MLP2 com predição direta utilizando as mesmas variáveis de entrada da MLP1 (sem a necessidade da altura total da árvore) ficou em torno de 0,01\%. O modelo MLP3 que utiliza como dados de entrada o $D A P$ e $H t$ ficou em torno de $0,30 \%$, já as diferenças percentuais para o modelo de Schumacher e Haal está em torno de 0,17\%. Em todos os casos observados a diferença percentual é menor que 1\%, mostrando que não há diferenças significativas e que todos os modelos foram capazes de calcular o volume real com boa margem de precisão.

Os erros de estimativa de volume foram calculados para cada classe e os resultados podem ser observados na Tabela (3). Os valores de MAE\% apresentados pela MLP1 variam de $4 \%$ a $6 \%$. O Bias $\%$ apresenta valores que variam de $0,24 \%$ a $1,69 \%$ que a princípio são bons valores, mas, podese observar que o Bias\% da classe 4 apresenta um valor elevado, como pode ser observado na Tabela (5), o volume calculado foi subestimado em relação ao valor real, Em boa parte das classes os valores de RMSE\% apresentam-se bastante satisfatórios e a correlação $R$ variou entre $0,95 \%$ 


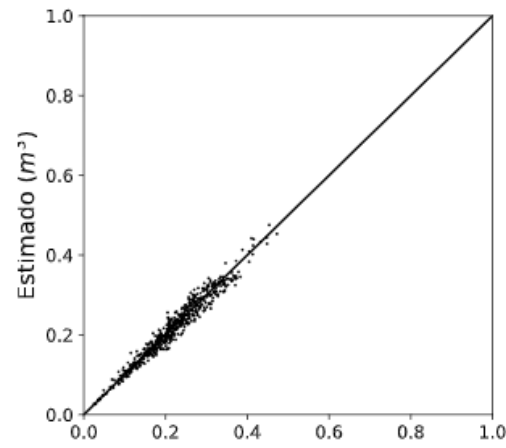

(a) Dispersão em MLP1

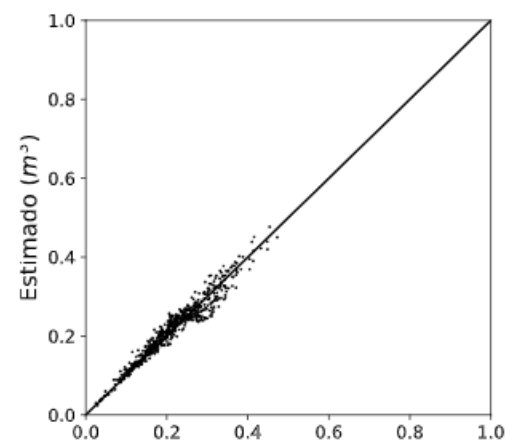

(b) Dispersão em MLP2

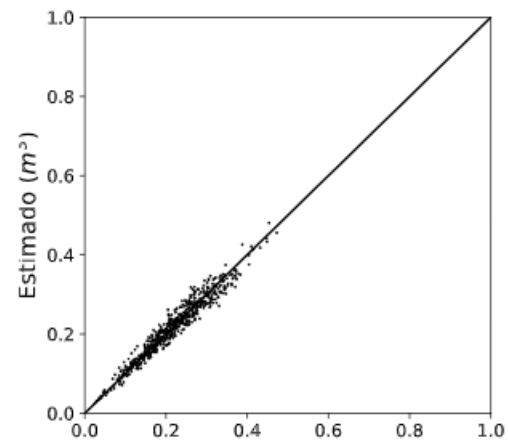

(c) Dispersão em MLP3

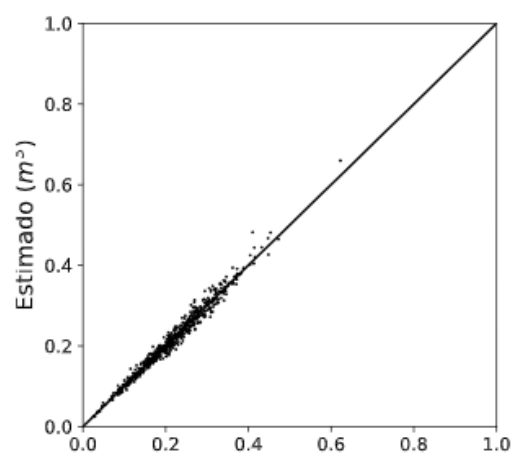

(d) Dispersão em Shumacher e Hall (log)

Figura 3. Dispersão entre os volumes reais e os volumes preditos pelas redes MLPs e Schumacher and Hall $(\log )$.

a $0,98 \%$, indicando que há uma forte correlação entre os volumes reais e estimados.

Os valores de MAE\% e RMSE\% da MLP2 e MLP3 mostraram-se pouco acima da MLP1 e de Schumacher e
Hall (log), ainda assim apresemtam-se como valores satisfatórios para o problema. As correlações $R$ de ambas arquiteturas variaram entre $0,77 \%$ a $0,96 \%$ para MLP2 e $0,87 \%$ a $0,97 \%$. Embora a classe 4 da MLP2 mostrouse uma correlação menor em relação aos demais modelos, pode-se verificar que em todos os modelos apresentaram bons resultados em comparação ao método de Schumacher e Hall (log).

As correlações menores nas classes 2, 3 e 4 dos volumes calculados principalmente pela MLP2 e MLP3 podem ser explicadas pelo fato de haver uma maior dispersão nos valores calculados. Essa dispersão pode ser observada na Figura (3).

A Figura 3(a) mostra o gráfico de dispersão entre o volume estimado pela MLP1 em relação aos volumes reais, a Figura 3(b) ilustra a dispersão dos volumes estimados pela MLP2 em relaão aos volumes reais, a Figura 3(c) mostra o gráfico da despersão entre os volumes estimados pela MLP3 e os valores reais, por fim, a Figura 3(c) mostra a dispersão dos volumes reais e estimados pelo método de Shumacher e Hall $(\log )$.

A ANOVA de 2-critérios foi realizada para os conjuntos de dados e apresentou como resultado o valor de $p=0,7827$. Este valor é maior que 0,05 indicando que a hipótese nula não pode ser rejeitada, implicando que não há diferenças estatísticas entre os modelos de MLPs e Schumacher e Hall $(\log )$.

\section{CONCLUSÕES}

Neste trabalho, foram apresentadas arquiteturas de redes neurais para estimar diâmetros e calcular o volume de clones de eucalipto. Foram utilizadas medidas nos diâmetros inicias das árvores em modelos com ou sem o conhecimento prévio da altura da árvore. As redes foram treinadas com o conjunto de $70 \%$ treino e $30 \%$ teste. As comparações com o método de Schumacher e Hall (log) mostrou que as redes conseguiram aproximar com bastante precisão os valores reais obtidos através da cubagem rigorosa. Os resultados obtidos através da MLP2 mostrou ser possível aproximar dos volumes reais sem a necessidade da altura total, uma medida crucial em muitos métodos de equações volumétricas além de ser uma medida onerosa de se obter em campo.

Os resultados mostraram-se satisfatórios para estimar volumes dos clones de eucalipto, tornando as arquiteturas utilizadas, recomendadas para a automação do inventário florestal reduzindo consideravelmente o tempo e custo deste processo.

\section{REFERÊNCIAS}

Bengio, Y., Goodfellow, I.J., and Courville, A. (2015). Deep learning. Nature, 521(7553), 436-444.

Bergstra, J. and Bengio, Y. (2012). Random search for hyper-parameter optimization. Journal of Machine Learning Research, 13(Feb), 281-305.

Binoti, M.L., Breda Binoti, D.H., Garcia Leite, H., Lages Ribeiro Garcia, S., Ferreira, M.Z., Rode, R., and Araújo Lopes da Silva, A. (2014). Redes neurais artificiais para estimação do volume de árvores. Revista Árvore, 38(2). 
Cabacinha, C.D. (2003). Um método para a realização do inventário florestal suprimindo a cubagem rigorosa. Ph.D. thesis, Universidade Federal de Lavras.

Diamantopoulou, M.J. (2005). Artificial neural networks as an alternative tool in pine bark volume estimation. Computers and electronics in agriculture, 48(3), 235244.

Diamantopoulou, M.J. (2010). Filling gaps in diameter measurements on standing tree boles in the urban forest of thessaloniki, greece. Environmental Modelling $\mathscr{G}$ Software, 25(12), 1857-1865.

Guo, F., Gao, G., Huang, J., Wang, L., and Wang, D. (2011). Diameter distribution prediction of populus shelterbelts based on artificial neural network. In $\mathrm{Na}$ tural Computation (ICNC), 2011 Seventh International Conference on, volume 2, 762-765. IEEE.

Huang, J., Zhao, J., Gao, G., Meng, X., and Guan, Y. (2009). Prediction of stand diameter distribution with artificial neural network. In Natural Computation, 2009. ICNC'09. Fifth International Conference on, volume 2, 79-82. IEEE.

IBÁ, I.R. (2017). Indústria brasileira de árvores.

Ji, Y., Hao, J., Reyhani, N., and Lendasse, A. (2005). Direct and recursive prediction of time series using mutual information selection. In International WorkConference on Artificial Neural Networks, 1010-1017. Springer.

Leduc, D.J., Matney, T.G., Belli, K.L., and Baldwin, V.C. (2001). Predicting diameter distributions of longleaf pine plantations: a comparison between artificial neural networks and other accepted methodologies. Res. Pap. SRS-25. Asheville, NC: US Department of Agriculture, Forest Service, Southern Research Station. $24 p ., 25$.

Marques da Silva, M.L., Breda Binoti, D.H., Gleriani, J.M., and Garcia Leite, H. (2009). Ajuste do modelo de schumacher e hall e aplicação de redes neurais artificiais para estimar volume de árvores de eucalipto. Revista Árvore, 33(6).

Niska, H., Skon, J.P., Packalen, P., Tokola, T., Maltamo, M., and Kolehmainen, M. (2010). Neural networks for the prediction of species-specific plot volumes using airborne laser scanning and aerial photographs. IEEE Transactions on Geoscience and Remote Sensing, 48(3), 1076-1085.

Nocedal, J. and Wright, S.J. (2006). Nonlinear Equations. Springer.

Özçelik, R., Diamantopoulou, M.J., Brooks, J.R., and Wiant Jr, H.V. (2010). Estimating tree bole volume using artificial neural network models for four species in turkey. Journal of environmental management, 91(3), $742-753$.

SCHUMACHER, F.X. (1933). Logarithmic expression of timber-tree volume. J Agric Res, 47, 719-734.

Shanno, D.F. (1970). Conditioning of quasi-newton methods for function minimization. Mathematics of computation, 24(111), 647-656.

Soares, F.A.A.M.N., Flores, E.L., Cabacinha, C.D., Carrijo, G.A., and Veiga, A.C.P. (2011a). Recursive diameter prediction and volume calculation of eucalyptus trees using multilayer perceptron networks. Computers and electronics in agriculture, 78(1), 19-27. doi:10.1016/ j.compag.2011.05.008.
Soares, F.A.A.M.N., Flores, E.L., Cabacinha, C.D., Carrijo, G.A., and Veiga, A.C.P. (2011b). Recursive diameter prediction and volume calculation of eucalyptus trees using multilayer perceptron networks. Computers and electronics in agriculture, 78(1), 19-27. doi:10.1016/ j.compag.2011.05.008.

Vieira, G.C., de Mendonça, A.R., da Silva, G.F., Zanetti, S.S., da Silva, M.M., and dos Santos, A.R. (2018). Prognoses of diameter and height of trees of eucalyptus using artificial intelligence. Science of The Total Environment, 619, 1473-1481.

Xiao, R., Carande, R., and Ghiglia, D. (1998). A neural network approach for tree height estimation using ifsar data. In Geoscience and Remote Sensing Symposium Proceedings, 1998. IGARSS'98. 1998 IEEE International, volume 3, 1565-1567. IEEE. 